\title{
The Effect of Sports Activity on Tibial Bone Strength in Junior High School Students
}

\author{
Masashi Watanabe*, Masaru Kaga $^{* *}$, Mutsumi Matsueda ${ }^{* *}$ and Kayo Takahashi** \\ ${ }^{*}$ Niimi College \\ 1263-2 Nishigata, Niimi, Okayama 718-8585 Japan \\ watanabe@niimi-c.ac.jp \\ **Faculty of Education, Okayama University \\ 3-1-1 Tushimanaka, Okayama-city, Okayama 700-8530 Japan \\ [Received December 7, 2006 ; Accepted April 18, 2007]
}

\begin{abstract}
The purpose of this study was to investigate the effect of sports activity on tibial bone strength in junior high school students. Tibial bone strength was evaluated by the speed of sound of tibia (t-SOS) using the Sound Scan $2000{ }^{\circledR}$. Thirty Male athletes, who participated in sports activities approximately 18 hours/week, were enrolled in this study. They were compared with 45 sedentary boys. t-SOS in athletes $(3707 \pm 67 \mathrm{~m} / \mathrm{sec})$ was significantly lower than that in sedentary boys $(3779 \pm 114 \mathrm{~m} / \mathrm{sec})$. Length of tibia in athletes $(40.2 \pm 2.1 \mathrm{~cm})$ was significantly longer than that in sedentary boys $(38.5 \pm$ $2.3 \mathrm{~cm})$. The value of t-SOS demonstrated a positive correlation with calcium intake from dairy products in athletes. These data suggest that sports activity and physique may affect tibial bone strength, and calcium intake may help to enhance tibial bone strength.
\end{abstract}

Keywords: sports activity, bone strength acquisition, junior high school athletes

[International Journal of Sport and Health Science Vol.5, 116-121, 2007]

\section{Introduction}

Children of junior high school age undergo significant somatic changes, as they are in their second period of rapid somatic growth, the first being the fetal period and infancy. Such children are also at the significant stage where long-axis bone development occurs, resulting in an increase in body length followed by a rapid increase in bone mass and bone strength (Boot, et al., 1997; Kaga, et al., 1999). Considering the difficulty of improving the deteriorated balance of resorption and formation of bone in elderly individuals, it seems useful to maximize the peak bone mass of children of junior high school age for the prevention of osteoporosis and fracture that usually occur later in life. According to a report by Mora, et al., (2003), children who are at risk of failing to achieve optimal peak bone mass during puberty require intervention for the purpose of improving their unhealthy lifestyles specifically to increase the amount of exercise and calcium intake to augment bone mass gain. Lifestyle during puberty can have a strong influence on the state of bone development.

Regarding factors that promote bone growth during childhood and adolescence, Thorsen, et al., (1999) have reported that the level of physical activity is one of the factors that affects bone mineral density. In addition, sufficient calcium intake has a beneficial effect on bone mass (Wallace, et al., 2002), and body weight correlates positively with bone mass (Lima, et al., 2001). In other words, factors enhancing bone growth include exercise, nutrition, and physical constitution, and the interaction of such factors determines the extent of bone growth. In Japan, since junior high school children tend to have more opportunities for organized sports activities than elementary school children due to the existence of sports club activities (Kaga, et al., 2004), an investigation of the effects of exercise on bone growth seems useful to optimize sports instruction for junior high school children. The majority of published reports on bone growth are based on the measurement of bone mass at the position where spongy bone reaches colossal proportions, such as the lumbar spine, radius, and calcanei. Few reports are based on the measurement of strengt $h$ of the long bones of the lower extremities, which are high in cortical bone that supports the load of body weight and physical exercise. Based on our investigation 
of tibial bone strength, therefore, we have reported that the bone strength of the sports group of ages 13-15 was lower than that of the control group not participating in sports (Watanabe, et al., 2003). In the same investigation, however, the body height of the sports group was significantly greater than that of the control group.

The present study aimed to clarify the effects of sports activities on tibial bone strength gain by comparing two groups of junior high school students with similar heights: a sports player group and a control group not participating in sports on a regular basis.

\section{Method}

\subsection{Subjects}

The subjects of this study were 30 male junior high school sports players (hereinafter referred to as the sports group) who had participated in sports activities on a daily basis over a prolonged period of time. Participating not only in their junior high school sports club activities but also in specially-arranged training and competitions as select athletes playing for their region, subjects spent an average of $18.6 \pm$ 6.2 hours per week on sports activities on an average of $6.7 \pm 0.4$ days per week. The average age of the sports group subjects was $14.3 \pm 0.5$ years. Eighteen subjects were soccer players, and twelve were soft tennis players. The control group consisted of 45 male students enrolled at T. Junior High School in O. City, who, with the exception of the physical education classes required by the school curriculum, had not participated in sports activities regularly. The average age of the control group was $14.4 \pm 0.5$ years. Since this study aimed at examining the relationship between bone strength and the length of participation in sports activities, age-related changes in height, weight, and length of tibia were not examined. All participants received an explanation of the goal and procedure of this study, and investigators obtained approval before initiating measurements and investigation.

\subsection{Measurements}

\subsubsection{Bone strength}

In this study, the tibial speed of sound (hereinafter referred to as t-SOS $\mathrm{m} / \mathrm{sec}$ ) was measured by a Sound
Scan 2000 Compact (Myriad Ultrasound System Ltd., Israel). T-SOS was measured as the peak value of transmission speed of an ultrasonic pulse along the cortical layer of the tibia by positioning a probe at the longitudinal midpoint of the tibia and then by moving it parallel to the long axis along the mid-tibial plane.

Length of tibia was measured according to the method required by the above- mentioned device: we placed subjects in the sitting position with the knee joint at an angle of 90 degrees and used a tape measure to manually measure the length between the upper end of the tibial tuberosity and the lower end of the malleolus medialis on the anterior surface of the tibia. The tibial tuberosity, projecting from just below the patella, is easily palpated with the subject' s knee joint positioned at 90 degrees. In their study on the relationship between length of tibia and t-SOS, Kaga, et al., (1999) measured the length of tibia with the same method. All measurements in this study were performed by the same examiner.

The Sound Scan 2000 Compact is mobile and poses no risk of radiation exposure. The amount of time required for measurement is as short as 3-5 minutes per subject, and the coefficient of variation for t-SOS (including variations caused by examiner and light conditions) is reported to be 0.24 (Foldes, et al., 1995). Therefore, we feel the Sound Scan 2000 Compact is suitable for assessing bone strength and screening for bone metabolic disorders in children and adolescents. The tibia is relatively straight, and one-third of its anterior surface is in immediate contact with the skin-that is, not enclosed in soft tissue. Due to the absence of soft tissue interference, this measurement method has a higher degree of reliability in comparison to other methods (Rosenthall, et al., 1996), and in 1998 it was the first ultrasonic measurement method approved by the FDA (US Food and Drug Administration approval code: p970026).

\subsubsection{Physical parameters}

Subjects' height, weight, and length of tibia were measured. To compare the bone growth process, the ratio of tibia length to body height was calculated. Human long bone growth proceeds in a specific sequence, occurring distally from the tibia to the femur, then to the spine during a period of body growth in height (Roche, 1974). A high tibia length to body height ratio indicates that peripheral long bones are growing. 


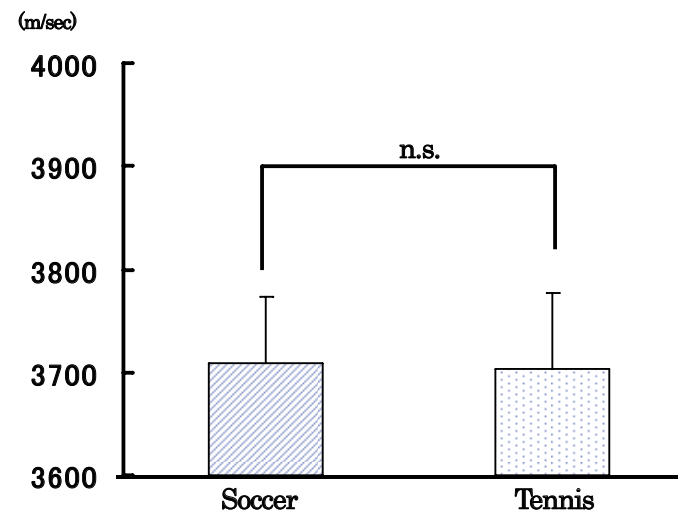

Figure 1 t-SOS values of soccer and tennis groups.

Table 1 Physical characteristics of the athletes

\begin{tabular}{lcccc}
\hline & $\mathrm{n}$ & Height $(\mathrm{cm})$ & Weight $(\mathrm{kg})$ & $\begin{array}{c}\text { Length of tibia } \\
(\mathrm{cm})\end{array}$ \\
\hline Soccer & 18 & $164.0 \pm 6.8$ & $51.2 \pm 7.3$ & $40.7 \pm 2.0$ \\
\hline Tennis & 12 & $161.0 \pm 8.3$ & $47.2 \pm 8.7$ & $39.5 \pm 2.2$ \\
\hline
\end{tabular}

Values are mean $\pm \mathrm{SD}$.

\subsubsection{Calcium intake from milk and other dairy products}

Using the intake of milk and other dairy products as indicators of calcium intake, we surveyed the weekly intake of milk and other dairy products through direct interview. In this survey, calcium intake from $200 \mathrm{cc}$ of milk was set as $200 \mathrm{mg}$, and calcium intake from one portion of dairy products, such as a slice of cheese or a cup of yogurt was set at as $130 \mathrm{mg}$.

\subsection{Statistical processing}

Statistical analysis in this study was performed by Stat View 5.0, and t-test was used for comparison between the sports group and the control group. Simple linear regression analysis was conducted to clarify the relationship between t-SOS and calcium intake. Statistical significance was set at $5 \%$ or less.

\section{Results}

\subsection{Bone strength measurement}

Figure 1 shows the t-SOS of the soccer players and soft tennis players in the sports group. The $\mathrm{t}-\mathrm{SOS}$ of the soccer players was $3709 \pm 65 \mathrm{~m} / \mathrm{sec}$, and that of the soft tennis players was $3703 \pm 73 \mathrm{~m} / \mathrm{sec}$;

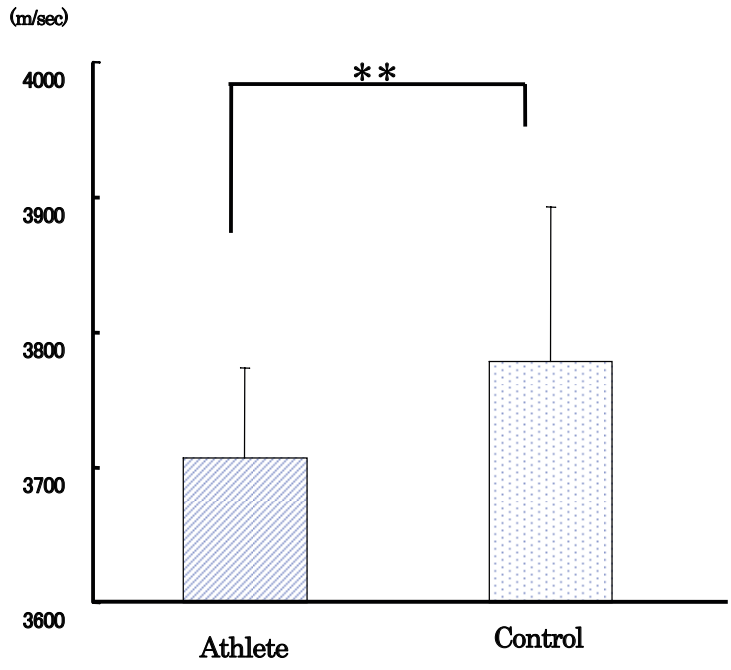

Figure 2 t-SOS values of athlete and control groups. $* * P<0.01$

Table 2 Physical characteristics of the subjects

\begin{tabular}{l|cccc}
\hline & $\mathrm{n}$ & Height $(\mathrm{cm})$ & Weight $(\mathrm{kg})$ & $\begin{array}{c}\text { Length of tibia } \\
(\mathrm{cm})\end{array}$ \\
\hline Athlete & 30 & $162.8 \pm 7.4$ & $49.6 \pm 8.0$ & $40.2 \pm 2.1 * *$ \\
Control & 45 & $160.9 \pm 6.7$ & $51.5 \pm 9.5$ & $38.5 \pm 2.3$ \\
\hline
\end{tabular}

Values are mean $\pm \mathrm{SD}$.

$* * p<0.01$

thus, we found no significant difference between these types of players.

Figure 2 shows the t-SOS of the sports group (soccer players and soft tennis players) and the control group. The t-SOS of the sports group overall was $3707 \pm 67 \mathrm{~m} / \mathrm{sec}$, significantly lower $(p<0.001)$ than that of the control group, which was $3779 \pm 114$ $\mathrm{m} / \mathrm{sec}$.

\subsection{Physical characteristics}

Table 1 shows the respective physical characteristics of the soccer players and soft tennis players. There was no significant difference in body height, weight, and length of tibia between these groups.

Table 2 shows the respective physical characteristics of the sports group and the control group. There was no significant difference in body height and weight between the two groups, but the average length of tibia of the sports group players was significantly higher $(p<0.01)$ than that of the control group. Also, the ratio of tibia length to body height of the sports group was significantly higher $(p<0.01)$ than that of the control group (Figure 3). 


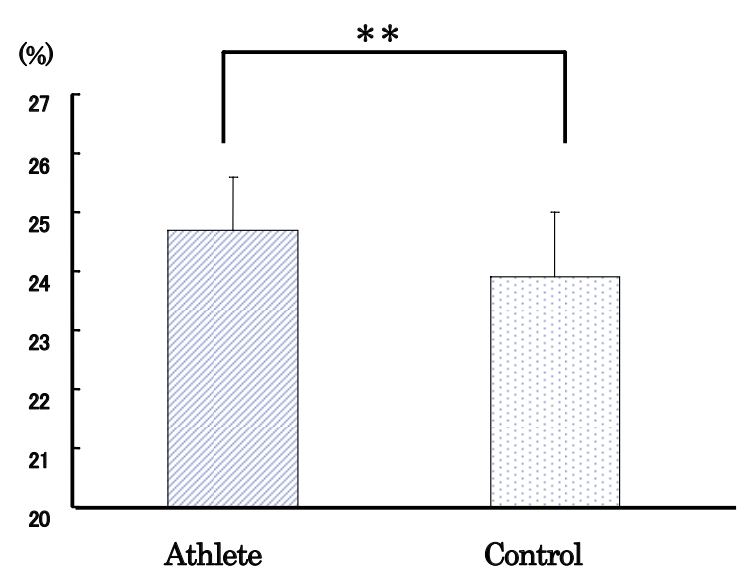

Figure 3 Length of tibia/ height values of athlete and control groups.

$* * P<0.01$

\subsection{Calcium intake}

Calcium intake from milk and other dairy products was $3795 \pm 1707 \mathrm{mg} /$ wk for the soccer players and $2957 \pm 1829 \mathrm{mg} / \mathrm{wk}$ for the soft tennis players, revealing that there was no significant difference between the two groups. Similarly, there was no significant difference in the calcium intake between the sports group $(3460 \pm 1775 \mathrm{mg} / \mathrm{wk})$ and the control group ( $3108 \pm 2185 \mathrm{mg} / \mathrm{wk})$. In the sports group, there was a positive correlation $(r=0.57, p<0.01)$ between $\mathrm{t}$-SOS and calcium intake from milk and other dairy products (Figure 4). In the control group, however, no such correlation was observed (Figure 5).

\section{Discussion}

Kaga, et al., (1999) have reported age-related changes in the t-SOS of Japanese children and adolescents. They found that bone strength increased with age, and there was a clear spurt at 14 years of age in males. Both groups in our study were within this period of rapid increase in bone strength, which is considered to be an important period in terms of bone strength gain. A beneficial effect of exercise on bone strength gain has been demonstrated in a study of forearm bone mass measurement in tennis players, which shows that the bone mass of the dominant arm is higher than that of the non-dominant arm (Ducher, et al., 2005). The sports group in our study consisted of players of soccer and soft tennis, which are thought to apply significant stress on the tibia which contributes to the promotion of bone formation.

The t-SOS of the sports group, however, was

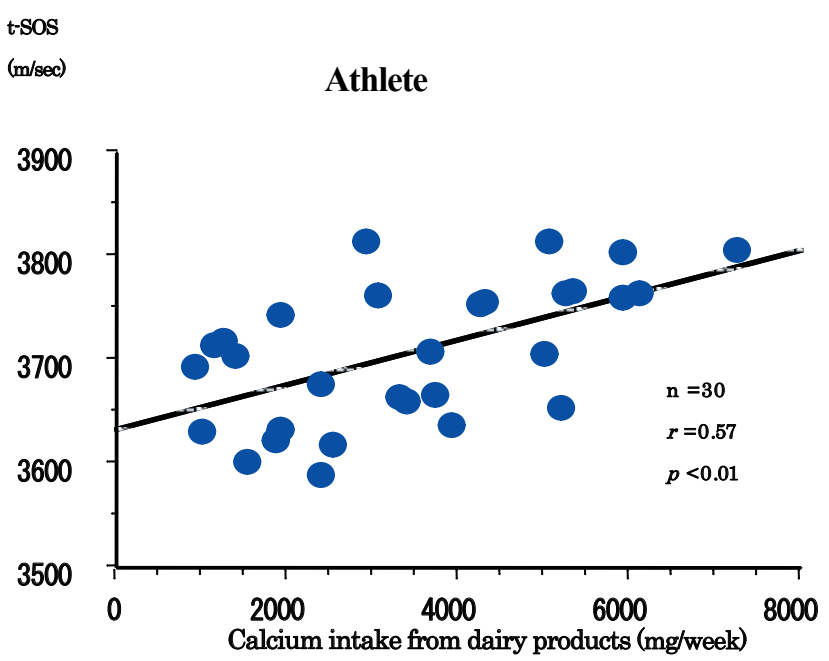

Figure 4 Relationship between t-SOS and calcium intake from dairy products.

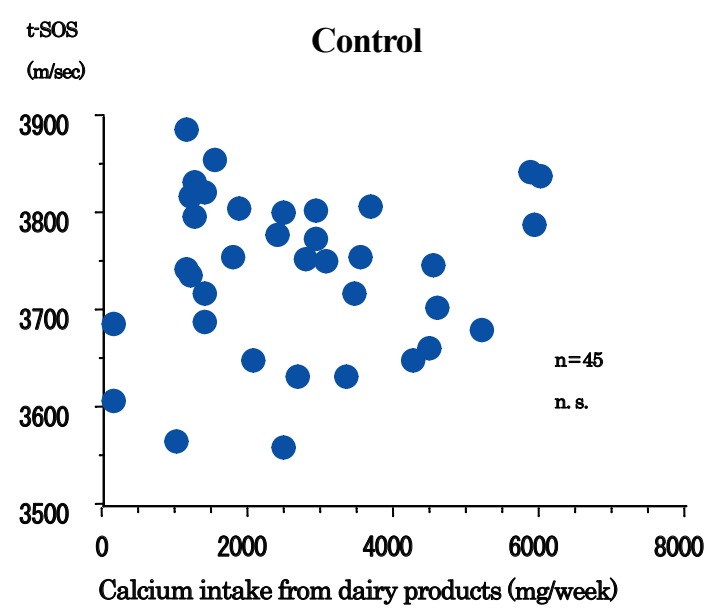

Figure 5 Relationship between t-SOS and calcium intake from dairy products.

significantly lower than that of the control group. This result can be associated with the physical constitutional characteristics of the sports group, whose tibia is longer and whose ratio of tibia length to body height is higher than that of the control group. While bone geometry relates to the frequency of daily exercise and impulse strength (Aki, et al., 2006), the relationship between increase in length of tibia and sports activity is not clear. Meanwhile, since there is an orderly sequence of long bone growth, which is equivalent to growth in body height, peripheral long bones are growing at a relatively early stage of bone growth when the ratio of tibia length to body height is significantly high (Roche, 1974), and during the time when body height increases rapidly, there is a period of relative bone fragility in which bone strength gain is delayed 
against an increase in length of the tibia (Kaga, et al., 2002). Judging from their physical characteristics, the sports group in this study was in a period of relative bone fragility.

The effects of exercise factors on bone growth need to be examined qualitatively and quantitatively. Though it seems difficult to precisely identify the qualitative aspect of exercise, the type of sport that one plays can serve as an index. For example, participants in sports activities performed on land, in which the full effect of gravity is experienced, exhibit higher bone mineral density and bone strength than individuals who engage in water sports, in which the effect of gravity is lower (Ozawa, 1994; Okano, et al., 2003). Frequency and length of participation are often used as an index to evaluate the quantitative aspects of sports activities. Based on the measurement of bone mineral densities of dancers that practiced for 13 hours per week vs. retired dancers that had practiced 8 hours per week prior to retirement, Valentino, et al., (2001) reported finding no difference in the bone mineral densities in the two groups. Bone formation of long-distance runners could be negatively affected by training, which is high in length and frequency (Komiya, 2001). These reports can be understood as negating positive correlations between the amount of sports activities and bone mass increase. Another report demonstrated that a period of body length increase is a period of modeling, in which the bone undergoes enlargement, as well as a period of rapid bone turnover (Parfitt, 1994). Taking this into consideration, junior high school can be viewed as a period when bone formation is apt to be affected by sports activities. The sports group in our study was engaged in such activities on an almost daily basis, for approximately 18 hours per week, which may be beyond the extent that the sports activities had a positive effect on t-SOS. Thus, we confirm that the effects of exercise on bone formation are associated not only with the type of sports activities but also with the quantitative aspects of frequency and length. As mentioned earlier, since the possibility that t-SOS was lower due to physical constitutional factors (e.g., the average length of the tibia in the sports group is longer than that in the control group), further examination will be necessary to clarify the relation ship between sports activities and bone strength for those who are at the same stage of tibia length development.
Regarding calcium intake and bone formation, Boot, et al., (1997) have reported that calcium intake positively correlates with bone mineral density; thus, calcium intake is important for bone formation. However, in the control group in our study no correlation was observed between t-SOS and calcium intake while a positive correlation between the two was observed in the sports group. We feel this is due to the higher calcium requirement of the sports group, whose members tend to lose more calcium through sweating. Therefore, we speculate that the effects of calcium intake on bone information are greater in junior high school sports players who participate in sports activities often compared to players who participate irregularly or less often. While calcium intake is important for bone formation in all junior high school children, the optimal amount of calcium should be determined according to the amount of physical activity.

\section{Conclusion}

In this study, the effects of sports activities on tibial bone strength gain have been examined utilizing t-SOS as an index. Tibial bone strength was assessed by t-SOS using a Sound Scan 2000 Compact. The subjects of this study were male junior high school students who participated in sports activities for $18.6 \pm 6.2$ hours per week, and a control group consisting of male students who participated in no regular sports activities except for physical education classes required by the school curriculum. The results of the investigation are as follows:

- The t-SOS $(3707 \pm 67 \mathrm{~m} / \mathrm{sec})$ of the junior high school sports players with a high frequency of sports activity was significantly lower than that of the control group $(3779 \pm 114 \mathrm{~m} / \mathrm{sec})$ of the same age that engaged in no regular sports activities.

- The tibia length of the junior high school sports players $(40.2 \pm 2.1 \mathrm{~cm})$ was significantly higher than that of the control group $(38.5 \pm 2.3 \mathrm{~cm})$.

- In the junior high school sports player group, calcium intake from milk and other dairy products positively correlated with t-SOS $(r=0.57, p<0.01)$.

Our results have confirmed that the amount of time devoted to participation in sports activities is directly related to the growing process of the length of tibia affect tibia bone strength gain during the 
period of junior high school. We also showed that calcium intake is important for junior high school sports players who participate in sports activities on a highly frequent basis. The effects of sports activities on bone strength can be further clarified by future investigations of the relationship between sports activities and bone strength in individuals at the same stage of growth.

\section{References}

Aki V., Raija K., Harri S., Erkki V., Juhani L., \& Timo J. (2007) Effect of impact exercise and its intensity on bone geometry at weight-bearing tibia and femur. Bone, 40: 604-611.

Boot, A.M., de Ridder, M.A., Pols, H.A., Krenning, E.P., \& de Muinch Keizer-Schrama, S.M. (1997) Bone mineral density in children and adolescents: relation to puberty, calcium intake, and physical activity,J Clin Endocrinol Metab,82(1): 57-62.

Ducher G., Jaffre C., Arlettaz A., Benhamou CL., \& Courteix D. (2005) Effects of long-term tennis playing on the muscle-bone relationship in the dominant and nondominant forearms. Can J Appl Physiol 30(1):3-17.

Kaga, M., Takahashi, K., Suzuki, H., Matsueda, M., Moriwake, T., Makino, H., Yamamoto, K. \& Seino Y. (1999) Standard values for ultrasonic measurement of the tibia in childhood and adolescence. J Jpn Soc Bone Morphom 9: 23-27.

Kaga, M., Takahashi, K., Suzuki, H., Matsueda, M., Moriwake, T., Miyatake, N. \& Seino, Y. (2002) Ultrasound assessment of tibial cortical bone acquisition in Japanese children and adolescents. J Bone Miner Metab 20:111-115.

Kaga, M., Takahashi, K., \& Seino, Y. (2004) Bipolarization of frequency of physical activity in childhood and adolescence. J Jpn Pediatr Soc 108: 625-634.

Komiya, Y. (2001) Study on the bone minerl density of female long-distance runners. J Clin Sports Med 9 (3): 328-331.

Lima, F., De Falco, V., Baima J., Carazzato, J.G. \& Pereira, R.M. (2001) Effect of impact load and active load on bone metabolism and body composition of adolescent athletes. Med Sci Sports Exerc 33(8): 1318-1323.

Mora, S. \& Gilsanz, V. (2003) Establishment of peak bone mass. Endocrinol Metab North Am 32(1): 39-63.

Ozawa, H. (1994) Exercise type and bone mineral density. J Clin Sports Med 11: 1245-1251.

Okano, R., Naka, S., Katsuki, K. \& Katsuki, M. (2003) The characteristics of the calcaneal bone stiffness and its relationships with the shapes and the fundamental physical fitness in the male athletes. J Clin Sports Med 20: 591-597.

Parfitt, A.M. (1994) The two faces of growth: benefits and risk to bone integrity. Osteoporosis Int 4: 382-398.

Roche, A.F. (1974) Differential timing of maximum length increments among bones within individuals. Human Biology 46: 145-157.

Rosenthall, L., Caminis, J. \& Tenenhouse, A . (1996) Correlation of ultrasound velocity in the tibial cortex, calcaneal ultrasonography, and bone mineral densitometry of the spine and femur. Calcif Tissue Int 58: 415-418.

Thorsen, K., Nordstrom, P., Lorentzon, R. \& Dahlen, G.H. (1999) The relation between bone mineral density, insulin-like growth factor I, lipoprotein (a), body composition, and muscle strength in adolescent males. J Clin Endocrinol Metab 84(9): 3025-3029.

Wallace, L.S. \& Ballard, J.E. (2002) Lifetime physical activity and calcium intake related to bone density in young women. $\mathrm{J}$ Womens Health Gend Based Med 11(4): 389-398.

Watanabe, M., Kaga, M., Suzuki, H. \& Takahashi, K. (2003) Influences of sports activity in junior high-school athletes on bone strength and fractures. Japanese journal of sport education studies 23 (2): 113-122.

Valentino R., Savastano S., Tommaselli A.P., D'Amore G., Dorato M., \& Lombardi G. (2001) The influence of intense ballet training on trabecular bone mass, hormone status, and gonadotropin structure in young women. J Clin Endocrinol Metab 86 (10): 4674-4678.

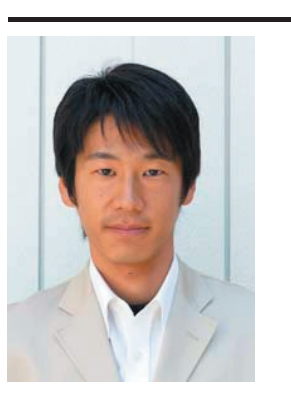

Name:
Masashi Watanabe

Affiliation:

Niimi College

Address:

1263-2 Nishigata, Niimi, Okayama 718-8585 Japan

Brief Biographical History:

2001- Master's Program, Graduate School of Education, Okayama University

2003- Joint Graduate School (Ph.D. Program) in the Science of School Education, Hyogo University of Teacher Education (PhD candidate)

2006- Research Associate, Niimi College

2007- Assistant Professor, Niimi College

Main Works:

- "Influences of sports activity in junior high-school athletes on bone strength and fractures.” Japanese Journal of Sport Education Studies 23 (2): 113-122 (2003)

- "The developmental changes and laterality of walking motion in infant." The Japanese Journal for the Education of Young Children 14: 1-7 (2005).

Membership in Learned Societies:

- Japan Society of Physical Education, Health and Sport Sciences

- Japanese Society of Sport Education

- Japanese Association of School Health

- The Japanese Society for the Education of Young Children

- The Japanese Society of Health Education of Children 